\title{
Two-phase flow expansion: development of an innovative test-rig for flow characterisation and CFD validation
}

\author{
Alberto Traverso, Federico Reggio*, Paolo Silvestri*,Sergio Rizzo, Geoff Engelbrecht, \\ Alexandros Chasoglou. \\ Thermochemical Power Group, DIME, University of Genoa, Via Montallegro 1, 16145, Genova, Italy
}

\begin{abstract}
The aim of this work is to describe the design of an innovative test rig for investigating the expansion of saturated fluids in the two-phase region. The experimental test rig was thought up and built by TPG of the University of Genoa. It will be equipped by probes and some optical accesses that permit high speed video recording and laser measurements. It will be useful for the study of the quality ratio, vapour and liquid droplet thermodynamic properties and their speed.
\end{abstract}

\section{Introduction}

Many thermodynamic cycles take advantage of the phase change in working fluid before the fluid expansion (e.g. steam and organic Rankine cycles or reverse cycles [1]). In case the expansion enters the saturation region, two-phase flow may occur with the formation of droplets, which can damage the expander blades. Experimental investigation concerning the expansion of saturated fluids can validate numerical models and contribute to the development of new turbo-expander prototypes less prone to erosion [2].

\section{The experimental test rig}

The test rig designed by Thermochemical Power Group (TPG) at the University of Genoa is composed by two stainless steel plates, both with a circular hole for the glass housing, which let optical access to the evolution of the flow (figures 1 and 2). Between the two plates there is a $1 \mathrm{~mm}$ stainless sheet, suitably cut, to create the fluid passages through 2 nozzles and an empty circular chamber between the two glasses (1 $\mathrm{mm}$ gap).

\footnotetext{
*Corresponding authors: federico.reggio@edu.unige.it, p.silvestri@unige.it
} 


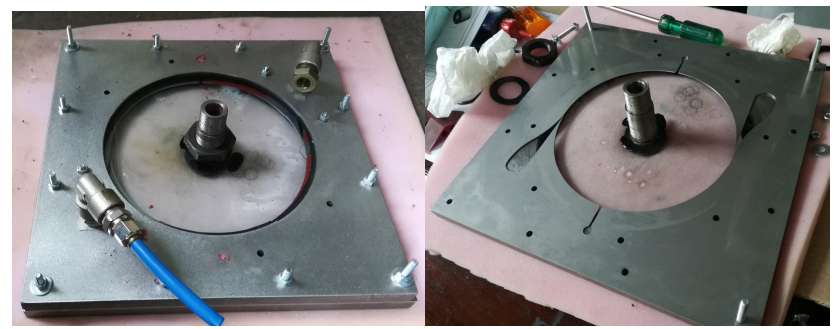

Figure 1 The complete test rig (left) and the rig opened (right)

For the experiments, saturated water (initial quality equal to 0 ) will be used at the absolute pressure of 4 bar and corresponding temperature of about $140^{\circ} \mathrm{C}$. The fluid enters through the two inlet holes (diameter: $1 / 2 \mathrm{inch}$ ) and a mass flow of 1.3-2 g/s will evolve inside each nozzle and in the gap between the two glasses, then it exits through a hollow shaft placed in the centre of the circular windows (figure 2). Two passages at the periphery are also present to discharge liquid accumulation in the chamber between the windows.

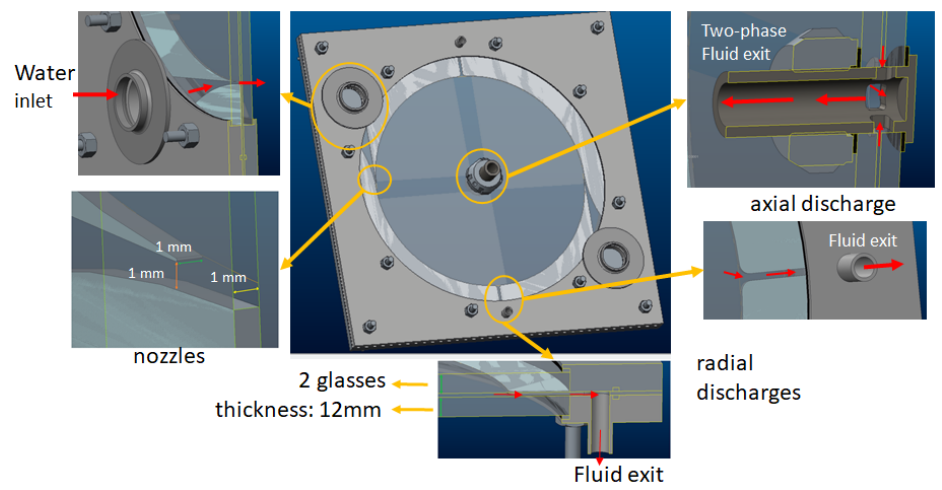

Figure 2 Test rig CAD 3D and fluid passages

To avoid window break, it has been chosen a tempered glass thickness of $12 \mathrm{~mm}$ and EPDM has been selected as sealing material thanks its low young modulus $(6 \mathrm{MPa})$ and its temperature resistance ( $\max$ working temperature of $150^{\circ} \mathrm{C}$ ).

Optimal pressure probe positions are chosen at the beginning, in the middle and at the tip of the nozzle; other pressure probes are placed in the chamber.

Ansaldo Energia Spa will provide University of Genoa with a high speed video-camera and will support for set up of Background-Oriented Schlieren (BOS) technique [3] to characterise the two-phase flow. BOS is an optical density visualization technique that uses correlation techniques on a background dot pattern to quantitatively characterize compressible and thermal flows with good spatial and temporal resolution.

Limmat Scientific Ag (LSC) developed and will provide an optical instrument for the test-rig able to measure the droplet size $(20-120 \mu \mathrm{m}$ in diameter) and speed. It employs forward scattering laser lights [4,5]: the amount of light scattered by droplets is proportional to the size of the droplet and the intensity of the incident light.

All the measurement results will be compared with those of the (CFD) numerical models developed by LSC and Aristotelio Panepistimio Thessalonikis.

\section{Conclusions}

This work describes an innovative test rig designed for the analysis of two-phase fluid expansion in a nozzle. The rig will be employed by TPG in a measurement campaign whose collected data will be useful for the design of a new resistant two-phase turbo expander. 
This project has received funding from the European Union's Horizon 2020 research and innovation programme under grant agreement No 764706, PUMP-HEAT project

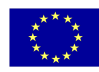
(https://www.pumpheat.eu/).

\section{References}

1. L. G. Hays, J. J. Brasz "Two-Phase Turbines for Compressor Energy Recovery" International Compressor Engineering Conference 1996

2. V. A. Tishchenkoa, R. A. Alekseeva, and I. Yu. Gavrilova," A Model of the Motion of Erosion-Hazardous Droplets in Steam Turbines' Interblade Channels" ISSN 00406015, Thermal Engineering, 2018, Vol. 65, No. 12, pp. 885-892.

3. M.Raffel," Background-oriented schlieren (BOS) techniques", Experiments in Fluids March 2015

4. H. Di, Z. Wang, D. Hua, "Precise size distribution measurement of aerosol particles and fog droplets in the open atmosphere", OPTICS EXPRESS A890 Vol. 27, No. 12 | 10 Jun 2019

5. F. Mahrt, J. Wieder, R. Dietlicher, H. R. Smith, C. Stopford, Z. A. Kanji," A highspeed particle phase discriminator (PPD-HS) for the classification of airborne particles, as tested in a continuous flow diffusion chamber" Atmos. Meas. Tech., 12, 3183-3208, 2019 